\title{
Criminologie
}

\section{Troubles mentaux et violence}

\section{Frédéric Millaud}

Volume 29, numéro 1, printemps 1996

Intervenir auprès des délinquants

URI : https://id.erudit.org/iderudit/017378ar

DOI : https://doi.org/10.7202/017378ar

Aller au sommaire du numéro

Éditeur(s)

Les Presses de l'Université de Montréal

ISSN

0316-0041 (imprimé)

1492-1367 (numérique)

Découvrir la revue

Citer cet article

Millaud, F. (1996). Troubles mentaux et violence. Criminologie, 29(1), 7-23.

https://doi.org/10.7202/017378ar

\section{Résumé de l'article}

Epidemiologic and clinical studies are presented of persons with mental disorders and a history of violent behavior. Knowledge of the risk factors associated with acts of violence committed by mentally disordered persons is essential for the initiation of preventive programs. The aim of this paper is therefore to introduce epidemiologic and clinical elements useful in assessing these risk factors. Furthermore, intervention strategies are described to deal with violence in mentally ill patients.
Ce document est protégé par la loi sur le droit d'auteur. L'utilisation des services d’Érudit (y compris la reproduction) est assujettie à sa politique d'utilisation que vous pouvez consulter en ligne.

https://apropos.erudit.org/fr/usagers/politique-dutilisation/ 
Epidemiologic and clinical studies are presented of persons with mental disorders and a history of violent behavior. Knowledge of the risk factors associated with acts of violence committed by mentally disordered persons is essential for the initiation of preventive programs. The aim of this paper is therefore to introduce epidemiologic and clinical elements useful in assessing these risk factors. Furthermore, intervention strategies are described to deal with violence in mentally ill patients.

\section{INTRODUCTION}

Ce texte est centré sur les problèmes de violence auxquels se heurtent les malades mentaux. On ne tiendra pas compte des individus dont certains traits de personnalité, voire certains troubles de personnalité, peuvent les amener à une incarcération (personnalités antisociales, par exemple). Par contre, les outils d'évaluation de dangerosité et les principes d'intervention auprès de patients violents, évoqués plus loin, peuvent dans une certaine mesure avoir un intérêt avec tous les types de populations violentes, qu'il s'agisse de malades mentaux ou non.

Les malades mentaux sont souvent associés à la violence dans la population générale et même dans les médias (Link, Cullen et Frank, 1987; Wahl, 1987). On peut dire que la stigmatisation dont souffrent les malades mentaux est certainement double en ce qu'elle est à la fois liée à la pathologie psychiatrique, à la folie en tant que telle, et, d'autre part, à la crainte que la folie peut susciter, en particulier la crainte de gestes violents qu'on ne peut pas prévenir, qui paraissent irraisonnés, immotivés. Sur le plan historique, on se rappellera que la psychiatrie à ses débuts s'est construite autour du "fou criminel» (Senninger, 1990). Après le XIX siècle, cependant, cette association systématique est écartée, ce qui a abouti à la séparation de la psychiatrie et de la criminologie. L'idée qui domine par la suite, tout au moins chez les cliniciens, est que les malades mentaux ne semblent pas plus dangereux que la population générale (Hafner et Böker, 1973; Guze, 1976).

1. L'auteur est médecin psychiatre et chef du département de psychiatrie de l'Institut Philippe-Pinel de Montréal, 10905, boul Henri-Bourassa Est, Montréal (Qc) H1C 1H1. Il est aussi professeur adjoint de clinique au département de psychiatrie de l'Université de Montréal. 
Dans un premier temps, nous donnerons quelques éléments sur l'évolution des idées en ce qui concerne la question de la prédiction de violence. En effet, cette préoccupation est devenue centrale au fil des ans et elle oriente toujours les recherches et les réflexions.

Nous indiquerons ensuite quelques données générales sur la violence des malades mentaux. Les études épidémiologiques portant sur de grands échantillons de population permettent d'identifier certains indicateurs de violence chez les malades mentaux en les comparant à la population générale. Ce type de travail peut déboucher sur des programmes de prévention et influencer certaines décisions sociales ou politiques. Elles sont donc d'un grand intérêt.

Toutefois, la portée générale de ces études épidémiologiques ne permet pas une intégration de l'ensemble des éléments qualitatifs propres à chaque individu. C'est pourquoi il est utile, pour le clinicien, de posséder également certains outils d'évaluation et de traitement pour faire face aux situations cliniques quotidiennes. Nous évoquerons alors les éléments retenus dans l'inventaire de dangerosité psychiatrique, outil élaboré àl'Institut Pinel de Montréal.

Dans une dernière partie, nous donnerons quelques principes d'interventions thérapeutiques tirés de notre expertise auprès de patients psychiatriques violents.

\section{PRÉDICTEURS DE VIOLENCE ET FACTEURS DE RISQUE}

Jusqu'au milieu des années 1980 , les psychiatres et psychologues ont été perçus comme totalement incapables de prédire les comportements violents (Monahan, 1981 ; Steadman, 1983). Par la suite, cependant, cette perception s'est un peu transformée et certaines études ont montré que la prédiction à court terme semblait un peu plus réalisable (Tardiff, 1989; Durivage, 1989; McNiel et Binder, 1991). Plus récemment, Apperson, Mulvey et Lidz (1993) ont démontré les défauts méthodologiques des premières études sur la prédiction clinique à court terme de comportements violents et Lidz, Mulvey et Gardner (1993) concluent que le jugement clinique a été sous-évalué dans les recherches précédentes. Il faut souligner que les cliniciens ne font pas franchement de prédiction de comportements violents, comme le soulignent Mulvey et Lidz dès 1988, mais abordent cette question de la dangerosité en termes de conditions contingentes.

Ainsi, les attentes créées par le désir de prédire la violence et l'impossibilité de le faire sans erreur ont amené chercheurs et cliniciens à prendre conscience de leurs capacités réelles, sans les minimiser, mais sans chercher non plus de recette infaillible. 
La question de la prédiction de violence a donc été reconceptualisée en termes d'identification de facteurs de risque de violence. L'introduction de la notion de risque laisse donc toujours une place à l'incertitude. En clinique, cette perspective nous paraît beaucoup plus appropriée. En effet, les patients psychiatriques sont soumis à des facteurs environnementaux qui interagissent avec leur personnalité et leur pathologie. Leur niveau de dangerosité psychiatrique varie en fonction de toute une série de facteurs. On peut donc affirmer que la dangerosité ou le risque de violence ne peut pas être établi une fois pour toutes; il existe des fluctuations dans le temps et des variations d'intensité. On se situe donc plus sur un continuum que dans un registre dichotomique. Cela implique également en clinique des révévaluations périodiques pour une prévention efficace. Cela amène aussi un certain rapprochement entre chercheurs et cliniciens. On retrouve ainsi dans la grande étude de MacArthur aux États-Unis cette prise en compte de la clinique (Steadman et coll., 1994, pp. 300-301): «Les facteurs de risque associés à la violence que nous explorons sont ceux qui, pensons-nous, jouent un rôle dans l'apparition de la violence chez les malades mentaux et que l'on peut évaluer sans trop de difficulté dans la pratique clinique actuelle; les facteurs de risque étudiés sont : (a) ceux associés à la violence dans des recherches antérieures; (b) ceux que nous croyons être associés à la violence de par l'expérience des cliniciens ${ }^{2}$... »

\section{DONNÉES GÉNÉRALES ET DONNÉES DES ÉTUDES ÉPIDÉMIOLOGIQUES LES PLUS RÉCENTES}

Nous tenterons dans cette partie de faire ressortir les points saillants des recherches effectuées sur les malades mentaux. Quels que soient les types de recherche (cliniques et épidémiologiques), certains éléments dominent la littérature et apparaissent comme les données générales actuellement valides. Nous les mentionnerons avant d'aborder les études épidémiologiques proprement dites.

\section{a) Les actes de violence des malades mentaux}

En ce qui concerne les homicides commis par les malades mentaux, il n'existe que peu d'études (Gottlieb, Gabrielsen et Kramp, 1987 ; Lindqvist, 1986; Taylor et Gunn, 1984). Les chiffres sont peu précis et oscillent entre 20 et $50 \%$ des homicides. Cependant, les facteurs d'intoxication par alcool ou drogues semblent prendre dans ces chiffres une place particulièrement grande et nous incitent donc à atténuer l'importance du rôle joué par les maladies mentales dans la commission des homicides. Par ailleurs, il est clair que la majorité des cas d'agression n'entraînent pas de blessures

2. C'est nous qui traduisons. 
graves et que ce sont surtout les patients récidivistes qui posent les gestes les plus graves (Haller et Deluty, 1988).

\section{b) Les victimes}

On constate que les victimes sont principalement (50 à $70 \%$ des cas) les membres de l'entourage familial (Addad et Benezech, 1977; Cormier et coll., 1971 ; Estroff et Zimmer, 1994 ; Millaud, 1989). Les mères paraissent le plus souvent visées dans les cas de violence répétée (Estroff et Zimmer, 1994). D'autre part, lorsqu'il s'agit de patients hospitalisés, les victimes deviennent alors soit les membres du personnel soignant, soit les autres patients. Cela confirme donc les indications données par Cormier dès 1971 qui mentionnait que les patients psychiatriques commettaient des agressions surtout dans le cadre de relations spécifiques. Autrement dit, ce sont les personnes qui sont en contact de façon privilégiée avec les malades mentaux et qui établissent certains liens sur un plan affectif qui sont principalement visées lorsqu'il y a agressions.

\section{c) Les éléments démographiques}

Sur le plan démographique, la plupart des études démontrent que les jeunes adultes sont plus violents que le reste de la population. En ce qui concerne les patients psychiatriques, il semble exister un risque plus élevé de violence chez les patients de moins de 30 ans (James et coll., 1990; Swanson, 1994). Les hommes paraissent plus violents que les femmes dans la plupart des études. Les proportions sont de l'ordre de neuf hommes pour une femme (Craig, 1982; Noble et Rodger, 1989; Tardiff et Sweillam, 1980). Les études cliniques vont généralement dans ce sens, même si certaines études très récentes nous indiquent une certaine modification de la répartition des hommes et des femmes dans les gestes de violence (Klassen et O'Connor, 1994 ; Steadman et coll., 1994). On trouve également plus de célibataires et de personnes de statut socio-économique faible (Smith et Hucker, 1991; Williams, Thorby et Sandlin, 1989).

\section{d) Les facteurs de risque identifiés dans les études épidémiologiques}

Il faut avant tout préciser que la notion de maladie mentale varie d'une étude à l'autre. Il n'y a pas d'uniformité. Cependant, même si les diagnostics de trouble de personnalité, d'abus d'alcool ou de drogues font partie des classifications diagnostiques usuelles (DSM-IV), ils ne sont pas considérés comme des maladies mentales dans la plupart des grandes études épidémiologiques; on distingue alors volontiers les maladies mentales majeures (schizophrénie, trouble affectif majeur, trouble paranoïaque et autres psychoses) des autres. 
À l'instar de Torrey (1994), on peut identifier cinq grands types d'études: (a) les études d'individus arrêtés; (b) les études de patients hospitalisés; (c) les études de patients traités en externe; (d) les études des familles avec un membre malade mental; (e) les études d'échantillons de la population générale.

Études des individus arrêtés. Depuis les années 1960 (époque de la désinstitutionnalisation), ces études montrent que le taux d'arrestations des malades mentaux est supérieur à celui de la population générale. Cependant, cela n'est pas un bon indice de violence, puisque la plupart des arrestations sont effectuées pour des délits non-violents (Torrey, 1994). Par ailleurs, certains auteurs ont examiné la proportion de malades mentaux parmi des populations de prisonniers mais sans s'attacher spécifiquement à l'identification d'éléments de violence. Ainsi, Jemelka, Trupin et Chiles (1989) nous indiquent que de 10 à $15 \%$ des prisonniers d'État aux ÉtatsUnis souffrent d'un trouble majeur de la pensée ou d'un trouble de l'humeur nécessitant des soins psychiatriques. Hodgins et Côté (1990) montrent qu'au Canada, la prévalence des troubles mentaux majeurs (schizophrénie, dépression majeure, troubles bipolaires) chez les populations pénitentiaires est supérieure à celle au sein de la population générale.

En ce qui concerne les crimes violents, Lamb et Grant (1982), à Los Angeles, ont montré que sur 96 prisonniers référés pour évaluation psychiatrique, $28 \%$ étaient accusés de crimes violents. Une étude similaire chez les femmes donne $18 \%$ de crimes violents. On soulignera cependant le biais introduit par le fait qu'il s'agit d'une population de prisonniers référés pour évaluation psychiatrique et non pas d'une sélection aléatoire sur une population carcérale globale. Une autre approche est l'examen de l'état mental des sujets ayant commis des crimes particuliers, par exemple les études citées plus haut sur les homicides. Martell et Dietz (1992) ont par ailleurs étudié une série d'individus qui ont poussé ou tenté de pousser quelqu'un devant la rame de métro à New York. Les malades mentaux sont responsables de la majorité des gestes de ce type. Mais là encore, comme pour les homicides, le rôle joué par les drogues et l'alcool est très important.

Études des patients hospitalisés. 11 existe de nombreuses êtudes de patients hospitalisés. Plusieurs s'attachent à démontrer les liens entre des éléments repérables avant une admission à l'hôpital et les comportements violents intra-hospitaliers, qu'il s'agisse de l'urgence, d'une unité d'admission ou ailleurs. Il en ressort certains éléments identifiés comme facteurs de risque :

- des antécédents de gestes violents (Blomhoff, Seim et Friis, 1990; McNiel et coll., 1988; Palmstierna et Wistedt, 1988, 1990; Tardiff, 1991). Cet élément est considéré comme le meilleur prédicteur de violence et toutes les études d'importance en tiennent compte; 
- le jeune âge, l'apparition récente de la maladie, l'angoisse (Kay, Wolkenfeld et Murrill, 1988);

- les abus d'alcool et de drogues (Kay, Wolkenfeld et Murrill, 1988; Palmstierna et Wistedt, 1988);

- la dépression, la manie (Kay, Wolkenfeld et Murrill, 1988; McNiel et Binder, 1989);

- les éléments d'organicité cérébrale (Convit et coll., 1988);

— les symptômes psychotiques (Humphreys et coll., 1992).

Par ailleurs, Tardiff (1991) signale qu'environ $10 \%$ des patients ont commis des gestes violents envers autrui juste avant leur admission. Si l'on inclut les bris d'objets et les agressions verbales, 35 à $45 \%$ des patients sont alors concernés. D'autres études décrivent les caractéristiques des patients violents hospitalisés en termes symptomatologiques et diagnostiques. Ainsi, sont le plus souvent documentés :

- les symptômes psychotiques, délire et hallucinations (Lowenstein, Binder et McNiel, 1990; Noble et Rodger, 1989; Tardiff, 1991);

- l'impulsivité (Apter, Plutchik, et Van Praag, 1993);

- l'angoisse (Apter et coll., 1993; Tardiff, 1991);

- certains diagnostics : schizophrénie (Krakowski, Convit et Volavka, 1988; Noble et Rodger, 1989; Palmstierna et Wisdedt, 1991), manie (Janofsky, Spears et Neubauer, 1988), dépression (Morrison, 1992).

Ces études présentent surtout un intérêt clinique et permettent de faire ressortir certains facteurs associés aux comportements violents des patients. Cependant, elles sont d'un intérêt limité, puisqu'il n'y a pas de comparaison avec la violence d'autres personnes non hospitalisées (Hodgins, 1993) et que les actes de violence sont un critère important pour l'hospitalisation psychiatrique (Torrey, 1994). Enfin, les interventions du personnel et les traitements influent sur le nombre d'actes violents commis par les patients (Torrey, 1994). Les études des patients qui sont sortis de l'hôpital semblent plus utiles (Torrey, 1994), même si elles ont parfois tendance à minimiser le problème. En effet, les patients ne sortent habituellement pas de l'hôpital tant qu'ils sont considérés comme potentiellement violents. Une revue des études précédant 1979 a été effectuée par Rabkin (1979) : il conclut que sur une période de 20 ans, les patients psychiatriques sortis de l'hôpital commettent plus de délits violents que la population générale. Une étude plus récente de Klassen et O'Connor (1990) mentionne que de 25 à $30 \%$ des patients psychiatriques masculins ayant des antécédents de violence deviennent à nouveau violents dans l'année qui suit leur départ de l'hôpital. Deux autres études portant plus spécifiquement sur les schizophrènes (Lindqvist et Allebeck, 1990; Shore, Filson et Rae, 1990) vont dans le même sens. L'étude de Lindquist et Allebeck, en particulier, ne présente 
que peu de défauts méthodologiques (Hodgins, 1993). À partir d'un échantillon de 644 schizophrènes suivis pendant quinze ans après leur première hospitalisation, cette étude montre qu'ils commettent quatre fois plus de crimes violents que la population générale. On relèvera cependant que les schizophrènes avec un dossier judiciaire ont aussi une histoire d'abus de drogues dans $55 \%$ des cas.

Études des patients traitês en externe. Elles sont complémentaires des précédentes (patients sortis de l'hôpital). Bartels et ses collaborateurs (1991) s'intéressent à un échantillon de 133 schizophrènes et mesurent leur agressivité à l'aide d'une échelle de 1 à 5 ( 1 pour l'agressivité verbale et 5 pour la violence physique avec lésions potentielles ou effectives). Les plus hauts niveaux d'agressivité sont corrélés de façon significative au sexe masculin, au diagnostic de trouble schizo-affectif ainsi qu'à l'absence de traitement pharmacologique. Soixante et onze pour cent des patients des niveaux 4 et 5 prennent mal la médication, contre $17 \%$ pour ceux qui ne manifestent aucune agressivité. Link et ses collaborateurs (1992) estiment que les patients psychiatriques ont deux à trois fois plus de risques de devenir violents. La seule variable qui les distingue de la population générale est le niveau des symptômes psychotiques; plus ils sont malades, plus ils risquent d'être violents.

Études des familles avec un membre malade mental. Toutes les études nous indiquent que c'est l'entourage familial qui est principalement visé lors des agressions. En 1990, la National Alliance for the Mentally Ill, aux États-Unis, a pratiqué une étude sur 1401 familles dont un membre est malade mental (schizophrénie, troubles bipolaires ou dépression majeure). Durant l'année précédant l'étude, $10,6 \%$ des patients ont blessé physiquement un membre de la famille et $12,2 \%$ ont menacé de le faire. On observe une différence entre les hommes et les femmes lorsqu'il s'agit de menaces d'agressions ( $25 \%$ d'hommes pour $12,5 \%$ de femmes) mais un score presque semblable pour les agressions ( $12 \%$ d'hommes et $9,5 \%$ de femmes).

Ces quatre premiers types d'études nous apportent des résultats intéressants en termes de données cliniques et descriptives. Ils permettent de faire certaines corrélations entre maladie mentale et violence et d'identifier certains facteurs de risque. Cependant, ces informations ne sont valables que dans le contexte spécifique de ces études et on ne peut en tirer des conclusions générales valides sur les liens entre maladie mentale et violence. Les études suivantes portant sur des échantillons de la population générale semblent plus intéressantes à ce niveau.

Études d'échantillons de population générale. Elles sont peu nombreuses, nécessitant des moyens économiques et humains souvent importants. Trois études sont à mentionner : celle effectuée par le National Institute of Mental Health et dont on nous rapporte deux séries de résultats 
(Robins et Regier, 1991; Swanson, 1994; Swanson et coll., 1990); une deuxième étude effectuée en Suède et rapportée par Hodgins (1992); la dernière menée par Link et Stueve (1994), laquelle s'attache plus spécialement au rôle des symptômes psychotiques dans les comportements violents. Sans entrer dans les détails de chaque étude, on dégagera les éléments les plus marquants. Si l'on sépare les troubles liés à l'utilisation de substances psycho-actives (alcool et drogues) des maladies mentales, on observe que :

$-85 \%$ des sujets violents ne sont pas des malades mentaux;

- $90 \%$ des sujets atteints de maladie psychiatrique grave ne sont pas violents (Swanson et Holzer, 1991).

Les malades mentaux asymptomatiques sans antécédents de violence se comparent à la population générale en termes de risque de violence.

Le risque de comportements violents chez les malades mentaux implique une symptomatologie aiguë.

L'abus de substances psycho-actives augmente le risque de violence chez les malades mentaux.

Il semble donc exister un sous-groupe de patients psychiatriques plus dangereux que la population générale dont on peut dégager quatre caractéristiques principales: (a) ces patients ont une histoire de violence antérieure; (b) ils n'observent pas la médication psychiatrique; (c) ils abusent d'alcool ou de drogues; (d) ils présentent une symptomatologie aiguë.

En ce qui concerne cette symptomatologie aiguë, certains symptômes psychotiques semblent plus significativement associés à la violence (Link et Stueve, 1994). Link et Stueve mentionnent en particulier que les patients de ce sous-groupe ont souvent l'impression que leur pensée est dominée par des forces hors de leur contrôle, que des pensées leur sont imposées de l'extérieur et qu'elles ne leur appartiennent pas et, enfin, qu'il y a des gens qui leur souhaitent du mal. Ils évoquent également le principe de la rationalité dans l'irrationalité pour expliquer certains comportements violents. Les patients psychotiques délirants sont convaincus de la réalité des éléments délirants qu'ils perçoivent et agissent donc de façon souvent rationnelle et congruente avec leurs perceptions et leurs convictions délirantes. Vus de l'extérieur, si on n'a pas accès à leur contenu mental, ces comportements paraissent alors imprévisibles, bizarres et irrationnels.

\section{L'ÉVALUATION DES FACTEURS DE RISQUE EN CLINIQUE}

Tout bon clinicien doit connaître les facteurs de risque de violence rapportés dans les recherches. Cependant, lorsqu'il s'agit de faire face à une situation clinique concrète, avec un patient donné, il est nécessaire d'avoir également en sa possession un outil d'évaluation, facile d'utilisation, 
complet et qui permette de prendre des décisions autres qu'intuitives ou «impressionnistes ». C'est dans cet esprit que les cliniciens de l'Institut Philippe-Pinel de Montréal ont élaboré un outil d'évaluation destiné spécifiquement à l'évaluation clinique des patients dangereux (Millaud et coll., 1992). Il s'agit d'un inventaire de dangerosité qui s'inspire en grande partie les indicateurs de risque de l'étude de MacArthur aux États-Unis (Steadman et coll., 1994). Nous ne reprendrons pas en détail cet inventaire de dangerosité car il a déjà fait l'objet d'une publication antérieure (Millaud et coll., 1992).

Rappelons cependant les principaux éléments qui le composent : (1) caractériser l'acte violent. Cette partie est fondamentale et doit être décrite avec le plus de précision possible. C'est une étape qui peut être difficile et demander du temps, mais elle est indispensable. On ne peut traiter de la même façon un meurtre et un fantasme d'agression, un acte isolé et un acte répétitif, etc.; (2) identifier les facteurs associés à l'acte violent. Il s'agit là d'identifier les facteurs de risque évoqués plus haut; on retrouve ainsi les facteurs démographiques, historiques, les facteurs d'intoxication par l'alcool ou les drogues, les éléments de perturbation de l'état mental (avec des parties symptomatologique et diagnostique). Ces éléments se retrouvent en grande partie dans les études épidémiologiques. Nous avons également tenu compte des aspects psychodynamiques, des facteurs environnementaux ou situationnels, des facteurs biologiques et enfin du niveau de reconnaissance du patient.

Les éléments psychodynamiques d'un patient doivent être dans la mesure du possible décodés. Identifier le noyau conflictuel principal d'un individu et les mécanismes de défense qu'il utilise est très utile en clinique pour mieux « comprendre » le patient. Autrement dit, commencer à donner un sens à l'acte violent, tenter de mettre des mots avec des patients qui ne mentalisent que très peu permet souvent de mettre en perspective les actes d'un patient et d'influencer les décisions thérapeutiques. Il s'agit d'une dimension couramment utilisée en clinique. Les facteurs environnementaux sont principalement les éléments extérieurs à l'individu et qui servent de déclencheurs (par exemple une séparation), de précipitants (par exemple la cessation d'un médication) ou d'éléments favorisants (par exemple les modèles de violence dans l'enfance).

Les facteurs biologiques sont très utiles pour préciser les diagnostics et les traitements pharmacologiques adoptés. Ils sont aussi à considérer pour tenter de déterminer quelles sont les capacités réelles du patient à contrôler son agressivité et ses capacités d'apprentissage. Un patient déficient ou porteur d'une lésion cérébrale n'aura pas les mêmes réactions ni les mêmes besoins thérapeutiques qu'un sujet d'intelligence normale ou sans lésion cérébrale objectivable. 
Enfin, le niveau de reconnaissance du patient face à sa violence est une question importante en clinique et qui est souvent d'un poids considérable dans les décisions thérapeutiques. Des mesures d'internement peuvent en découler en particulier. On doit tenter de qualifier le niveau de reconnaissance : d'une part, évaluer si le patient reconnaît la violence de son acte ou de ses fantasmes et dans quelle mesure il s'en attribue la responsabilité et, d'autre part, s'il connaît des signes précurseurs à l'acte violent. En effet, on observe souvent une série de petits signes qui paraissent s'enchaîner jusqu'au passage à l'acte. Par exemple, l'apparition d'une insomnie, une augmentation de l'anxiété qui peut se traduire par le besoin de marcher sans arrêt, une irritabilité verbale, etc.

L'utilisation d'un outil systématisé tel que l'inventaire de dangerosité permet avant tout de bien documenter la situation clinique sans occulter certaines parties. Elle permet donc de diminuer les problèmes liés à une surévaluation ou une sous-évaluation de la dangerosité ou, en d'autres termes, de réduire les réactions contre-transférentielles que suscitent les patients violents.

La diversité et le nombre d'éléments qui composent cet inventaire indiquent par ailleurs le niveau de complexité d'une telle évaluation et on ne doit pas hésiter, dans les situations les plus difficiles, à obtenir l'avis d'un ou plusieurs collègues. On verra d'ailleurs, dans la partie sur l'intervention, que le travail avec des patients violents se fait dans le cadre d'une équipe multidisciplinaire. Il s'agit là certainement de l'une des fonctions d'une telle équipe.

\section{QUELQUES PRINCIPES D'INTERVENTION AUPRÈS DES PATIENTS VIOLENTS}

L'intervention doit tenir compte de la complexité des situations cliniques dans lesquelles nous amènent jes patients violents. Cela suppose nécessairement une approche multidisciplinaire (Maden, 1993) et un travai] effectué en vue de la prévention de la violence (Millaud et coll., 1992; Rice, Harris et Quinsey, 1994). L'investigation clinique qui vise à documenter les éléments cités plus haut nous paraît la base essentielle à la mise en place d'un traitement. En effet, les zones à risque ainsi découvertes seront aussi les zones de travail à effectuer en collaboration avec le patient.

La première étape du traitement est donc l'évaluation clinique et, progressivement, le partage avec le patient des résultats de cette évaluation; à ce point, on se doit de communiquer au patient quels sont, selon nous, les différents éléments qui constituent des facteurs de risque de violence pour lui. Il doit en effet apprendre petit à petit à les connaître, à les accepter. Tant que les patients persistent à dévier leur violence et leurs facteurs de risque, il peuvent représenter un danger important. 
Ces patients, comme les autres patients psychiatriques, peuvent tirer profit de certaines interventions pharmacologiques. Certains médicaments peuvent aider à diminuer les manifestations d'agressivité (neuroleptiques, lithium, anticonvulsivants). L'utilisation de ces médications varie en fonction des psychopathologies rencontrées. Par ailleurs, des interventions psychothérapiques sont également utiles. Ces interventions ne s'effectueront qu'en l'absence de crise aiguë et relèveront pour la plupart d'un registre cognitivo-comportemental. Les approches groupales sont le plus souvent privilégiées, mais peuvent se jumeler avec des prises en charge individuelles. Lion et Tardiff (1987) ont suggéré quelques principes de base. Premièrement, on évaluera la motivation et les raisons du patient à s'engager dans un processus psychothérapique; deuxièmement, le patient doit développer un autocontrôle de ses émotions et de ses comportements, en particulier ses comportements violents et agressifs. Troisièmement, la verbalisation doit être encouragée, plutôt que les actes. Ceci présente une importante difficulté pour les patients violents qui ont de grandes difficultés à mentaliser et à verbaliser leurs émotions.

Des interventions familiales sont aussi fréquemment utiles. En effet, les patients violents engendrent souvent de la peur et de l'anxiété chez les autres membres de leur famille; en outre, il n'est pas rare que des dynamiques familiales violentes existent chez plusieurs membres de la famille, ce qui contribue à entretenir chez le patient ce type de comportements.

On doit tenir compte de certaines considérations légales avec de tels patients, en particulier les mesures d'internement en milieu psychiatrique (garde en établissement). Par ailleurs, des mesures de judiciarisation de certains actes violents peuvent être privilégiées selon les circonstances et les pathologies.

Enfin, en dehors de ces interventions thérapeutiques, il est nécessaire que tous les cliniciens respectent certaines règles spécifiques pour entrer en relation avec des patients violents et instaurer un cadre thérapeutique efficace et sécuritaire.

Premièrement, les interventions auprès de ces patients ne doivent être effectuées que si le clinicien se sent en sécurité sur le plan physique. On ne peut pas intervenir de façon appropriée si on est terrorisé. On se doit donc de ne pas chercher à intervenir et traiter les patients violents à tout prix, dans n'importe quelle condition.

Deuxièmement, une fois la sécurité du clinicien assurée, on doit informer le patient de l'évaluation faite à son sujet, des mesures qui sont prises à son égard, mesures qui doivent être congruentes avec l'évaluation de sa dangerosité. On doit, autant que possible, lui préciser ce qu'on attend de lui sur le plan clinique, sur le plan de ses comportements et de sa façon d'entrer en relation avec nous. 
Troisièmement, il est important d'établir des limites claires quant à ce qui est toléré et ce qui est interdit sur le plan de l'agressivité. Ces patients ont souvent tendance à transgresser les limites établies. Il faut les leur rappeler chaque fois qu'il y a transgression.

Il est important que les décisions concernant le management de ces patients soient prises en équipe de façon à ce que les éléments négatifs vécus par le patient ne soient pas identifiés à une seule personne qui pourrait alors servir de bouc émissaire et même qui pourrait être en danger.

Quatrièmement, il est important d'identifier avec le patient et de lui nommer les éléments de souffrance cernés par les membres de l'équipe et qui sont sous-jacents aux manifestations de violence.

Cinquièmement, les éléments de clivage dans lesquels nous amènent de façon systématique les patients violents, doivent être repérés et discutés entre les différents cliniciens. Cette situation est normale quand elle est transitoire. Dans la mesure où elle est identifiée, elle peut être alors facilement remise dans le contexte clinique propre du patient. La compréhension de ce phénomène de clivage doit alors être communiquée au patient et des mesures concrètes, congruentes et constructives peuvent alors être prises. Cela évite au patient de projeter à l'extérieur ses problèmes et de les faire porter par les autres, en particulier les soignants.

Sixièmement, le plan d'intervention doit être clair et facile d'application, de façon à ce que chacun des intervenants de l'équipe multidisciplinaire soit à l'aise et puisse appliquer le plan de soins sans difficulté.

On voit que la philosophie qui sous-tend l'ensemble de ces interventions est celle de l'écoute du patient, d'interventions actives, mais aussi celle de la responsabilisation.

En fonction des niveaux de violence des patients, des niveaux de risque et de l'évolution de ces facteurs au cours des traitements, les patients violents peuvent ainsi bénéficier de cadres de soins différents qui vont des soins externes à des soins hospitaliers, y compris ceux d'un hôpital sécuritaire comme l'Institut Philippe-Pinel de Montréal.

\section{CONCLUSION}

Il est intéressant de constater que depuis quelques années, l'intérêt des chercheurs et des cliniciens pour la question de la violence et de la dangerosité des malades mentaux s'est accru. De là une réflexion et des méthodes de recherche de plus en plus spécifiques. On constate par ailleurs une évolution des pratiques psychiatriques et plus généralement des pratiques cliniques, avec des dimensions d'encadrement légal de plus en plus rigoureuses. Cette réalité est un argument de plus pour préciser les risques qu'encourent personnellement nos patients et ceux qu'ils font courir aux 
autres. Cliniciens et chercheurs doivent donc continuer à unir leurs efforts dans ce domaine, lequel retrouve une place importante au sein de la clinique quotidienne.

\section{BIBLIOGRAPHIE}

ADDAD, M. et BENEZECH, M. (1977), « Schizophrénie et déliquance. Enquête médico-sociale chez 116 psychotiques, dont 83 délinquants, hospitalisés en centre hospitalier spécialisé », Annales médicales, vol. 1, $\mathrm{n}^{\circ}$ 1, pp. 1-33.

APPERSON, L., MULVEY, E. et LIDZ, C. (1993), "Short term clinical prediction of assaultive behavior: artifacts of research methods", American Journal of Psychiatry, vol. 150, pp. 1374-1379.

APTER, A., PLUTCHIK, R. et VAN PRAAG, H. M. (1993), «Anxiety, impulsivity and depressed mood in relation to suicidal and violent behavior», Acta Psychiatrica Scandinavica, vol. 87, $\mathrm{n}^{\circ}$ 1, pp. 1-5.

BARTELS, J., DRAKE, R. E., WALLACH, M. A. et coll. (1991), "Characteristic hostility in schizophrenic outpatients", Schizophrenia Bulletin, vol. 17, pp. 163171.

BLOMHOFF, S., SEIM, S. et FRIJS, S. (1990), «Can prediction of violence among psychiatric patients be improved?", Hospital \& Community Psychiatry, vol. 41, $\mathrm{n}^{\circ} 7$, pp. 77i-775.

CONVIT, A. et coll. (1988), «Predicting assaultiveness in psychiatric inpatients: A pilot study ", Hospital \& Community Psychiatry, vol. 39, $\mathrm{n}^{\circ} 4$, pp. 429-434.

CORMIER, B. et coll. (1971), «The psychodynamics of homicide in a specific relationship", Revue canadienne de criminologie, vol. 13, pp. 1-8.

CRAIG, T. J. (1982), «An epidemiological study of problems associated with violence among psychiatric inpatients", American Journal of Psychiatry, vol. 139, pp. 1262-1266.

DURIVAGE, A. (1989), "Assaultive behaviour : before it happens", Canadian Journal of Psychiatry, vol. 34, $\mathrm{n}^{\circ} 5, \mathrm{pp} .393-397$.

ESTROFF, S. E. et ZIMMER, C. (1994), « The influence of social networks and social support on violence by persons with serious mental illness », Hospital and Community Psychiatry, vol. 45, $\mathrm{n}^{\circ} 7, \mathrm{pp} .669-679$.

ESTROFF, S. E. et ZIMMER, C. (1994), "Social networks, social support and violence among persons with severe persistent mental illness », dans Monahan J., Steadman H., Violence and mental disorder, Monahan J. et Steadman H. (dir.), The University of Chicago Press, pp. 259-295.

GOTTLIEB, P., GABRIELSEN, G. et KRAMP, P. (1987), « Psychotic homicides in Copenhagen from 1959 to 1988 ». Acta Psychiatrica Scandinavica, vol. 76, pp. 285-292.

GUZE, S. (1976), Criminality and psychiatric disorders, New York, Oxford University Press, pp. 35-36.

HAFNER, H. et BÖKER, W. (1973), "Crimes of violence by mentally abnormal offenders, Cambridge, Cambridge University Press. 
HALLER, R. M. et DELUTY, R. H., "Characteristics of psychiatric inpatients who assault staff severely ", Journal of Nervous and Mental Disease, vol. 178, $n^{\circ} 8$, pp. 536-537.

HODGINS, S. et CôTÉ, G. (1990), «The prevalence of mental disorders among penjtentiary inmates », Canadian Mental Health, vol. 38, pp. 1-5.

HODGINS, S. (1992), « Mental disorder, intellectual deficiency and crime ", Archives of General Psychiatry, vol. 49, pp. 476-483.

HODGINS, S. (1993), "Criminality of Mentally Disordered Persons» dans Mental disorder and crime, Hodgins S. Éditeur, Sage Publications, pp. 3-21.

HUMPHREYS, M. S., JOHNSTONE, E. C., MACMILLAN, J. F. et TAYLOR, P. J. (1992), "Dangerous behaviour preceding first admissions for schizophrenia", British Journal of Psychiatry, vol. 161, pp. 501-505.

JAMES, D. V. et coll. (1990), "An increase in violence on an acute psychiatric ward : A study of associated factors ", British Journal of Psychiatry, vol. 156, pp. 846852.

JANOFSKY, J., SPEARS, S. et NEUBAUER, D. (1988), "Psychiatrist's accuracy in predicting violent behavior on an inpatient unit ", Hospital and Community Psychiatry, vol. 39, $\mathrm{n}^{\circ}$ 10, pp. 1090-1094.

JEMELKA, R., TRUPIN, E. et CHILES, J. A. (1989), "The mentally ill in prisons", Hospital and Community Psychiatry, vol. 40, pp. 481-485.

KAY, S. R., WOLKENFELD, F. et MURRILL, L. M. (1988), «Profiles of aggression among psychiatric patients: Covariates and predictors ", Journal of Nervous \& Mental Disease, vol. 176, $n^{\circ}$ 9, pp. 547-557.

KLASSEN, D. et O'CONNOR, W. (1990), «Predicting violence in schizophrenic and non-schizophrenic patients: A prospective study », Journal of Community Psychology, vol. $16, \mathrm{n}^{\circ} 2, \mathrm{pp} .217 .227$.

KLASSEN, D. et O'CONNOR, W. (1994), « Demographic and case history variables in risk assessment », in Violence and Mental Disorder, Monahan J. et Steadman H. (dir.), Chicago, The University of Chicago Press, pp. 229-257.

KRAKOWSKI, M. I., CONVIT, A. et VOLAVKA, J. (1988), "Patterns of inpatient assaultiveness : Effect of neurological impairment and deviant family environment on response to treatment ", Neuropsychiatry Neuropsychology and Behavioral Neurology, vol. 1, $\mathrm{n}^{\circ} 1$, pp. 21-29.

LAMB, H. R. et GRANT, R. W. (1982), "The mentally ill in an urban county jail», Archives of General Psychiatry, vol. 39, pp. 17-22.

LIDZ, C., MULVEY, E et GARDNER, W. (1993), "The accuracy of predictions of violence to others", Journal of the American Association, vol. 269, pp. 1007 1011.

LJNDQVIST, P. (1986), «Criminal homicide in Northern Sweden 1970-1981 : Alcohol intoxication, alcohol abuse and mental disease », International Journal of Law and Psychiatry, vol. 8, pp. 19-37.

LINDQVIST, P. et ALLEBECK. P., «Schizophrenia and crime. A longitudinal followup of 644 schizophrenics in Stockholm », British Journal of Psychiatry, vol. 157, pp. 345-350.

LINK, B. G., CULLEN, F. et FRANK, J. (1987), "The social rejection of former mental patients : understanding why labels matter», American Journal of Sociology, vol. 92, pp. 1461-1500. 
LINK, B. G., ANDREWS, H. et CULLEN, F. T. (1992), « The violent and illegal behavior of mental patients reconsidered", American Sociological Review, vol. 57, pp. 275-292.

LINK, B. G. et STUEVE, A. (1994), «Psychotic symptoms and the violent/illegal behavior of mental patients compared to community controls $\gg$, Violence and Mental disorder, Monahan J. et Steadman H. (dir.), The University of Chicago Press, pp. 137-159.

LION, J. et TARDIFF, K. (1987), « The long term treatment of the violent patient ", Psychiatry Update : Annual Review of the American Psychiatric Assocition, Hales R. et Frances A. (dir.), American Psychiatric Press, Washington DC, vol. 6, pp. 537-548.

LOWENSTEIN, M., BINDER, R. L. et MCNIEL, D. E. (1990), «The relationship between admission symptoms and hospital assaults», Hospital and Community Psychiatry, vol. 41, n 3, pp. 311-313.

MADEN, T. (1993), «The psychiatric management of violence», Violence : Basic and Clinical Science, Édition Thompson et Cowen, pp. 135-146.

MARTELL, D. A. et DIETZ, P. E. (1992), «Mentally disordered offenders who push or attempt to push victims onto subway tracks in New Youk City ", Archives of General Psychiatry, vol. 49, pp. 472-475.

McNIEL, D. E. et coll. (1988), « Predictors of violence in civilly committed psychiatric patients ", American Journal of Psychiarry, vol. 145, pp. 965-970.

McNIEL, D. E. et BINDER, R. L. (1989), «Relationship between preadmission threats and later violent behavior by acute psychiatric inpatients », Hospital and Community Psychiatry vol. $40, n^{\circ} 6$, pp. 605-608.

McNIEL, D. E. et BINDER, R. L. ( 1991), «Clinical assessment of the risk of violence among psychiatric inpatients", American Journal of Psychiatry, vol 148, $\mathrm{n}^{\circ} 10$, pp. 1317-1321.

MILLAUD, F. (1989), "L'homicide chez le patient psychotique : Une étude de 24 cas en vue d'une prédiction à court terme ", Revue canadienne de psychiatrie, vol. 34, pp. 340-346.

MILLAUD, F., ROY, R., GENDRON, P. et AUBUT, J. (1992), «Un inventaire pour l'évaluation de la dangerosité des patients psychiatriques ", Revue canadienne de psychiatrie, vol. 37, pp. 608-615.

MONAHAN, J. (1981), The clinical prediction of violent behavior, Washington DC, Governement Printing Office.

MORRISON, E. (1992), "A hierarchy of aggressive and violent behaviors among psychiatric inpatients», Hospital and Community Psychiatry, vol. 43, $\mathrm{n}^{\circ} 5$, pp. 505-506.

MULVEY, E. et LIDZ, C. (1988), «What clinicians talk about when assessing dangerousness? ", conférence à The Biennal Meeting of the American Psychology Law Society, Miami.

NOBLE, P. et RODGER, S. (1989), "Violence by psychiatric inpatients", British Journal of Psychiatry, vol. 155, pp. 384-390.

PALMSTIERNA, T. et WISTEDT, B. (1988), «Prevalence of risk factors for aggressive behavior: Characteristics of an involuntarity admitted population », Acta Psychiatrica Scandinavica, vol. 78, n 2, pp. 227-229. 
PALMSTIERNA, T. et WISDEDT, B. (1990), «Risk factors for aggressive behaviour are of limited value in predicting the violent behaviour of acute involuntarily admitted patients 》, Acta Psychiatrica Scandinavica, vol. 81, $\mathrm{n}^{\circ} 2$, pp. 152-155.

PALMSTIERNA, T. et WISDEDT, B. (1991), "The relationship of crowding and aggressive behavior on a psychiatric intensive care unit ". Hospital and Community Psychiatry, vol. 42, $\mathrm{n}^{\circ} 12$, pp. 1237-1240.

RABKIN, J. (1979), «Criminal behavior of discharged mental patients : A critical appraisal of the research ", Psychological Bulletin, vol. 86, pp. 1-27.

RICE, M, HARRIS, G. et QUINSEY, V. (1994), "Control in the psychiatric setting adults ", Handbook of aggressive and destructive behavior in psychiatric patients, Hersen M., Ammerman R et Sission L. (dir.), New York, Plenum Press, pp. 129143.

ROBINS, L. et REGIER, D. (1991), "Psychiatric Disorders in America: the Epidemiologic Catchment Area Study», New York, Free Press.

SENNINGER, J. L. (1990), "Dangerosité. Étude historique ", L'Information psychiatrique, vol. 7, pp. 690-696.

SHORE, D., FILSON, C. R. et RAE, D. S. (1990), «Violent crime arrest rates of White House case subjects and matched control subjects ", American Journal of Psychiatry, vol. 147, $\mathrm{n}^{\circ} 6$, pp. 746-750.

SMITH, J. E. et HUCKER, S. J. (1991), "Violence, current opinion", Psychiatry, vol. 4, pp. 841-845.

STEADMAN, H. (1983), "Predicting dangerousness among the mentally ill : Art magic and science», International Journal of Law and Psychiatry, vol. 6, pp. 381390.

STEADMAN, H., MONAHAN, J., APPELBAUM, P., GRISSO, T., MULVEY, E., ROTH, L., ROBBINS, P. et KLASSEN, D. (1994), «Designing a new generation of risk assessment research ", Violence and Mental disorder, Monahan et Steadman (dir.), The University of Chicago Press, pp. 297-318.

SWANSON, J. et coll. (1990), "Violence and Psychiatric Disorder in the Community: Evidence from the Epidemiologic Catchment Area Study », Hospital and Community Psychiatry, vol. 41, $\mathrm{n}^{\circ} 7$, pp. 761-770.

SWANSON, J. et HOLZER. C. (1991), "Violence and ECA data (letter to the editor)", Hospital and Community Psychiarry, vol. 42, pp. 954-955.

SWANSON, J. (1994), «Mental disorder, substance abuse, and community violence : an epidemiological approach », Violence and mental disorder, Monahan et Steadman (dir.), The University of Chicago Press, pp. 101-136.

TARDIFF, K. et SWEILLAM, A. (1980), «Assault, suicide and mental illness », American Journal of Psychiatry, vol. 37, pp. 164-169.

TARDIFF, K. (1989), A model for the short-term prediction of violence potential in current approaches to the prediction of violence, Washington DC, American Psychiatric Press Inc., pp. 3-12.

TARDIFF, K. (1991), "Violence by psychiatric patients ", Review of Clinical Psychiatry and the Law, pp. 175-233.

TAYLOR, P. J. et GUNN, J. (1984), "Risk of violence among psychotic men », British Medical Journal, vol. 288, pp. 1945-1949.

TORREY, E. F. (1994), «Violent behavior by individuals with serious mental illness », Hospital and Community Psychiatry, vol. 45, $\mathrm{n}^{\circ} 7$, pp. 653-662. 
WAHL, O. (1987), «Public vs professional conceptions of schizophrenic », Journal of Community Psychology, vol. 15, pp. 285-291.

WILLIAMS, W., THORBY, J. et SANDLIN, P. D. (1989), « Perceptions of prehospital dangerous behavior by psychiatric inpatients and their families", Journal of Psychiatry and the Law, vol. 17, $\mathrm{n}^{\circ} 1$, pp. 21-27. 